\title{
The Application of Improved Genetic Algorithm in the Markov State Transition Matrix to SME Technical Efficiency and Scale Revenue Calculation Methods
}

\author{
Wei Chuan-li ${ }^{\mathbf{1}, \mathbf{2}}$ \\ ${ }^{1}$ School of Economic and Management, Harbin Engineering University, Nantong \\ Str. 145, Harbin 150001, China \\ ${ }^{2}$ Schools of Public Finance and Administration, Harbin Commercial University, \\ Tongda Str. 138, Harbin 150028, China \\ weichuanli1975@163.com
}

\begin{abstract}
To solve a state transfer matrix using a Markov model, an improved genetic algorithm approach is proposed. A Markov prediction model is employed to study structures in the SME technical efficiency and scale gains calculation method. The obtained results are compared to the data shown in the China Statistical Yearbooks. It is found that our proposed genetic algorithm approach provides references for optimizing an agriculture SME industrial structure and hence improves the prediction precision.
\end{abstract}

Keywords: SME technical efficiency returns to scale Improved genetic algorithm

\section{Introduction}

SMEs are not only economic and social development of an important force, but also China's market economy the most dynamic part. The advantage of SMEs small and fine, small and specialized, small and live, in carrying out technological innovation activities has incomparable advantages of large enterprises, more than $75 \%$ of independent innovation products, more than $80 \%$ of patents are completed SMEs . China's foreign trade import and export volume of about $60 \%$ is provided by the SME, SME export trade to the rapid development of the national economy plays a huge role in promoting. Therefore, strengthening support for SMEs and nurturing for the sustainable development of the national economy has important significance.

As modern prediction approaches are with high scientificity, accuracy and applicability, Markov prediction methods are widely employed in economic and natural science fields. The success of applying these approaches is based on the full information on the mutual transfer probabilities of the possible states of predicted objects. To accurately predict the transfer probability matrix of the Markov states, it is required to enhance the precision of the Markov prediction. Existing methods for solving the transfer probability matrix of the states mainly include statistical estimate approaches, linear system equations approaches, multivariate regression approaches and quadratic programming approaches. Each type of methods has its own advantages and disadvantages. For examples, 1) statistical discrepancies exist and data obtaining procedures are complicated; 2) not all desired transfer probabilities of the states are guaranteed to be satisfied the condition $0 \leq \mathrm{Pij} \leq 1$; 3) all obtained regressive equations have to take a significant test. When the significant test is failed, other methods for solving the probability are required and solving the matrix becomes more complicated; and 4) a large amount of calculations is required and the solving process is complicated.

Genetic algorithms (GA) have been drawn a great attention in the artificial intelligence community since the middle of 1980s. At the early 1990s, it is widely studied in the 
computing science, information science and optimization communities. Also, it has become one of the crucial technologies for the intelligence computation since the 21st century. Its main advantages include that it is with whole searching strategy, the searches do not require the gradient information and the algorithms do not require continuously differentiable objective functions. The algorithms only require computing the objective functions. Hence, these methods are particularly applicable for solving optimization problems in complicated systems with highly nonlinear, discrete objective functions. Compare to existing methods, this paper proposes an improved genetic algorithm and employed for solving the transfer probability matrix of the states in the Markov model. The obtained results show that the proposed approach is a practical method for achieving high prediction accuracies.

\section{Markov Prediction Method}

Markov prediction method is to determine the change of the future trend of various states using the Markov chain. It is a stochastic prediction method via exploiting the transfer probabilities of the states of system objects. Therefore, it is a simple method for predicting the event probability. The basic idea is to obtain the transfer probability matrix of the states using raw data sequences, and then estimate the change of the future trend based on the transfer probability matrix of the states. In a dynamical system with random variables, the change of each state is called the state transition. Due to the variable nature of the transfer probabilities of different states of the system, the Markov method is more suitable for short term predictions.

\subsection{Markov Process}

The main title (on the first page) should begin $13 / 16$ inches ( 7 picas) from the top edge of the page, centered, and in Times New Roman 14-point, boldface type. Capitalize the first letter of nouns, pronouns, verbs, adjectives, and adverbs; do not capitalize articles, coordinate conjunctions, or prepositions (unless the title begins with such a word). Please initially capitalize only the first word in other titles, including section titles and first, second, and third-order headings (for example, "Titles and headings" — as in these guidelines). Leave two blank lines after the title.

\subsection{Transfer Probability Matrix of the States}

Let the $\mathrm{n}$ states of a system be $\mathrm{S} 1, \mathrm{~S} 2, \ldots, \mathrm{Si}, \ldots, \mathrm{Sn}$. For each time, the system could only stay in one of the states $\mathrm{Si} \quad(\mathrm{i}=1,2, \cdots, \mathrm{n})$. Suppose that the system is in the present state $\mathrm{Si}$. Denote the probability of transferring to the next state $\mathrm{Sj}$ as Pij. After the time $\Delta \mathrm{t}$, let the number of the transferring possibilities of $\mathrm{Si}$ be $\mathrm{n}$. The table below shows the transferring probabilities of the states.

Table1. Probabilities of System State Transition

\begin{tabular}{ccccc}
\hline State & $\mathbf{S}_{\mathbf{1}}$ & $\mathbf{S}_{\mathbf{2}}$ & $\ldots$ & $\mathbf{S}_{\mathbf{n}}$ \\
\hline $\mathbf{S}_{\mathbf{1}}$ & $P_{11}$ & $P_{12}$ & $\ldots$ & $P_{1 \mathrm{n}}$ \\
$\mathbf{S}_{\mathbf{2}}$ & $P_{21}$ & $P_{22}$ & $\ldots$ & $P_{2 \mathrm{n}}$ \\
$\vdots$ & $\vdots$ & $\vdots$ & $\vdots$ & $\vdots$ \\
$\mathbf{S}_{\mathbf{n}}$ & $P_{\mathrm{n} 1}$ & $P_{\mathrm{n} 1}$ & $\ldots$ & $P_{\mathrm{nn}}$ \\
\hline
\end{tabular}

These system states are mutually transferable in certain conditions and the matrix formed by the transferring these probabilities among the states is called the transfer probability matrix of the states. These transitions form a $\mathrm{n} \times \mathrm{n}$ transfer probability matrix 
and it is denoted as P. Hence, the one step transfer probability matrix P can be expressed as:

$$
P=\left[\begin{array}{cccc}
P_{11} & P_{12} & \cdots & P_{1 n} \\
P_{21} & P_{22} & \cdots & P_{2 n} \\
\vdots & \vdots & \vdots & \vdots \\
P_{n 1} & P_{n 2} & \cdots & P_{n n}
\end{array}\right]
$$

If the system states undergo a number of transitions rather than a single step transition, then it can be described by the $\mathrm{k}$ step transition matrix which is written as $\mathrm{P}(\mathrm{k})$. Thus, we have:

$\mathrm{P}(\mathrm{k})=\mathrm{P}(\mathrm{k}-1) \mathrm{P}=\mathrm{Pk}$

The $\mathrm{k}$ step transition matrix is used for characterizing the transitions where more than one transitions are involved on the basis of the previous transition matrix.

\subsection{Markov Prediction Model}

Assume that the number of possible states is $\mathrm{n}$ in a system. Also, the states are mutually exclusive. The initial state vector of the system $\mathrm{S} 1, \mathrm{~S} 2 \ldots \mathrm{Sn}$, is denoted as:

$\mathrm{S}(0)=(\mathrm{S} 1(0), \mathrm{S} 2(0), \ldots, \mathrm{Sn}(0)$

Here, $\mathrm{Si}(0)$ stands for the initial probability of the system in the state i. Since the probability of the system in the state $\mathrm{i}$ after the $\mathrm{k}$ step transitions is $\mathrm{Si}(\mathrm{K})$, the state vector after the $\mathrm{K}$ step transitions is:

$\mathrm{S}(\mathrm{k})=(\mathrm{S} 1(\mathrm{k}), \mathrm{S} 2(\mathrm{k}), \ldots, \mathrm{Sn}(\mathrm{k}))$

The relationship between $\mathrm{Si}(0)$ and $\mathrm{Si}(\mathrm{k})$ is:

$$
S(k)=S(k-1) P=S(0) P^{k}=S(0)\left[\begin{array}{cccc}
P_{11} & P_{12} & \cdots & P_{1 n} \\
P_{21} & P_{22} & \cdots & P_{2 n} \\
\vdots & \vdots & \vdots & \vdots \\
P_{n 1} & P_{n 2} & \cdots & P_{n n}
\end{array}\right]^{k}
$$

When the initial state $S(0)$ and the one step transition probability matrix $P$ are known, (1) can be applied to predict the system state at the time instant k. Hence, (1) is called the Markov prediction model. The key point of employing the Markov prediction model is based on the full information of $\mathrm{P}$ and the system initial state vector $\mathrm{S}(0)$.

\subsection{Method for Determining the Transfer Probability of the Markov States}

According to the principle of the least square method, let the transfer probability matrix of the estimated states be

$$
P^{\prime}=\left[\begin{array}{llll}
P_{11}^{\prime} & P_{12}^{\prime} & P_{13}^{\prime} & P_{14}^{\prime} \\
P_{21}^{\prime} & P_{22}^{\prime} & P_{23}^{\prime} & P_{24}^{\prime} \\
P_{31}^{\prime} & P_{32}^{\prime} & P_{33}^{\prime} & P_{34}^{\prime} \\
P_{41}^{\prime} & P_{42}^{\prime} & P_{43}^{\prime} & P_{44}^{\prime}
\end{array}\right]
$$

Let the fitting error at the time instant $\mathrm{t}$ of the system state $\mathrm{Sj}(\mathrm{j}=1,2, \ldots, \mathrm{n})$ be:

$$
e_{j}(t)=S_{j}(t)-\sum_{i=1}^{n} P_{i j}^{\prime} S_{i}(t-1) \quad(j=1,2, \cdots, n)
$$

Within the whole observation period $\mathrm{T}$, let the total square fitting error of the system state $\mathrm{Sj}$ be Qj:

$$
Q_{j}=\sum_{i=1}^{T}\left[e_{j}(t)\right]^{2} \quad(j=1,2, \cdots, n)
$$

Let the total square fitting error of the system state in the whole observation period be Q. Then, we have: 


$$
Q=\sum_{j=1}^{n} \sum_{t=1}^{T}\left[e_{j}(t)\right]^{2}
$$

The objective is to minimize the total square fitting error of the system state in the whole observation period. Let the obtained value of the transfer probability matrix of the state be ${ }^{P_{i j}^{\prime}}(\mathrm{i}=1,2, \ldots, \mathrm{n}, \mathrm{j}=1,2, \ldots, \mathrm{n})$. Then, we have the following nonlinear programming model:

$$
\begin{aligned}
& \min Q=\sum_{j=1}^{n} \sum_{t=1}^{T}\left[S_{j}(t)-\sum_{i=1}^{n} P_{i j}^{\prime} S_{i}(t-1)\right]^{2} \\
& \text { s.t. }\left\{\begin{array}{l}
\sum_{j=1}^{n} P_{i j}^{\prime}=1, \quad i=1,2, \cdots, n \\
0 \leq P_{i j}^{\prime} \leq 1, \quad i, j=1,2, \cdots, n
\end{array}\right.
\end{aligned}
$$

By solving the above nonlinear programming problem, the transfer probability matrix of the states $\mathrm{P}^{\prime}$ is obtained.

\section{Our Proposed Improved Genetic Algorithm}

The improved genetic algorithm is based on a binary encoding approach. From the evolution strategy viewpoint, our proposed improved genetic algorithm combines the parent individuals and the child generation individuals produced by crossover together to form a new temporary population and calculate the fitness value of each individual in the new temporary population. The $m$ individuals with the highest fitness values are reserved and the $\mathrm{m}$ individuals with the lowest fitness values are mutated. Then, the later $\mathrm{m}$ mutants are combined with the previously reserved $\mathrm{m}$ individuals to form a new temporary population. By sorting the new population, the $\mathrm{m}$ individuals with the highest fitness values are selected and the evolution of the population is completed. 


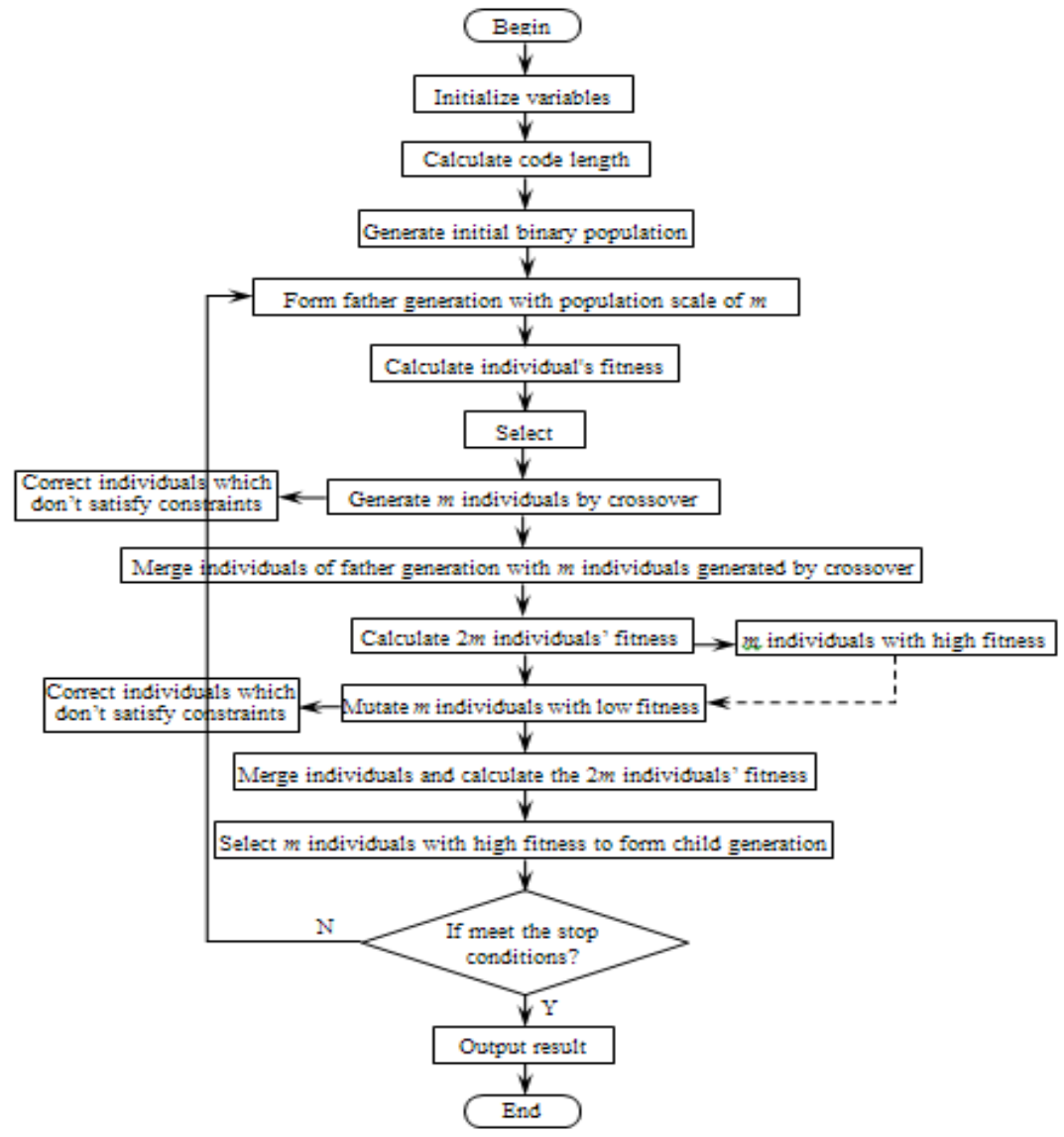

\section{Figure 1. Elite Group Keeping Genetic Algorithm Evolution Strategy Program Block Diagram}

Base on elitism reservation methods found in previous literatures, this method results to good qualities of population. Meanwhile, the excellent individuals are reserved among the individuals in the parent population and the individuals produced by crossover and mutation. Our proposed method can successfully prevent the occurrence of poor individuals generated by the crossover and mutation operations during the evolution process. In the mutation operation, the $m$ individuals with the lowest fitness values among the parent and the child generations produced by the crossover operation are mutated. In such method, the occurrence of the poor individuals is avoided while the mutation probability is increased. The proposed method enables to produce more new individuals in which it increases the population diversity, enhances the population periodicity and enable the escape from the local optimal solutions.

\section{Computer Numerical Simulation Results}

The section takes the structure of the SMEs agriculture industry in the Heilongiiang province as an example to illustrate the proposed method. The structure of the agriculture industry involves the constitution of all sections inside the agriculture industry as well as their interrelationships. It actually includes the structures of the industry and internal relations among the agriculture, forestry, animal husbandry and fishery industries. Heilongjiang province is one of the most important agricultural provinces in China. Its advantageous agricultural production conditions together with the scientific forecast of the proportional values of the provincial agriculture industry will benefit the province. This will also facilitate the rapid and sustainable developments of both the agriculture industry and rural economy. Furthermore, it provides a theoretical support for making 
rational decisions on adjusting the structure of the agriculture industry in the province. By adopting the Markov theory to the forecast of the structural problems arising in the agriculture industry, the agriculture, forestry, animal husbandry and fishery industries are connected each others. Also, by using the proposed improved genetic algorithm to solve the transfer matrix of the states in the Markov model, the calculation is much simpler and the obtained result is more accurate.

The data of the agriculture, forestry, animal husbandry and fishery industries of SMEs in the Heilongjiang province between 2005 and 2013 are presented in the China Statistical Yearbooks are summarized in Table 2.

Table 2. Agricultural Production Values of Various Industries in Heilongjiang Province Unit (Billion Yuan)

\begin{tabular}{ccccc}
\hline Year & agriculture & forestry & animal husbandry & fishery \\
\hline 2005 & 502.9 & 59.1 & 294.2 & 23.1 \\
2006 & 620.2 & 65.8 & 400.7 & 25.0 \\
2007 & 718.6 & 67.3 & 461.2 & 27.4 \\
2008 & 787.4 & 68.0 & 480.7 & 30.1 \\
2009 & 971.9 & 79.0 & 585.0 & 25.1 \\
2010 & 1142.3 & 89.6 & 813.1 & 36.0 \\
2011 & 1206.8 & 85.2 & 870.2 & 45.2 \\
2012 & 1369.2 & 95.5 & 965.8 & 53.7 \\
2013 & 1801.8 & 110.2 & 1189.9 & 58.9 \\
\hline
\end{tabular}

The proportional values of the agriculture, forestry, animal husbandry and fishery industries in the Heilongjiang province are calculated based on the data listed in Table 2 are presented in Table 3.

Table 3. Agricultural Production Value Proportion of Various Industries in Heilongjiang Province

\begin{tabular}{ccccc}
\hline Year & agriculture & forestry & animal husbandry & Fishery \\
\hline 2005 & 0.5720 & 0.0672 & 0.3345 & 0.0263 \\
2006 & 0.5579 & 0.0592 & 0.3604 & 0.0225 \\
2007 & 0.5638 & 0.0528 & 0.3618 & 0.0215 \\
2008 & 0.5764 & 0.0498 & 0.3518 & 0.0220 \\
2009 & 0.5851 & 0.0476 & 0.3522 & 0.0151 \\
2010 & 0.5489 & 0.0431 & 0.3907 & 0.0173 \\
2011 & 0.5467 & 0.0386 & 0.3942 & 0.0205 \\
2012 & 0.5511 & 0.0385 & 0.3888 & 0.0216 \\
2013 & 0.5700 & 0.0349 & 0.3765 & 0.0186 \\
\hline
\end{tabular}

Based on the data listed in Table 3, take the annual proportional values of the agriculture, forestry, animal husbandry and fishery industries in the Heilongjiang province as the state and the total square fitting error of the system state in the whole observation period (There are 8 years between 2006 and 2013.) as the optimization objective. The objective function is as follows:

$$
\min Q=\sum_{j=1}^{4} \sum_{t=1}^{8}\left[S_{j}(t)-\sum_{i=1}^{4} p_{i j}^{\prime} S_{i}(t-1)\right]^{2}
$$




$$
\text { s.t. }\left\{\begin{array}{l}
\sum_{j=1}^{4} p_{i j}^{\prime}=1, \quad i=1,2,3,4 \\
0 \leq p_{i j}^{\prime} \leq 1, \quad i, j=1,2,3,4
\end{array}\right.
$$

Based on the proposed improved genetic algorithm method, the nonlinear programming problem in (6) is solved. That is, Q is minimized and the transfer probability matrix of the state $\mathrm{P}^{\prime}$ is obtained. The parameters of the improved genetic algorithm are selected based on the following. The intervals of the variables are selected as $[0,1]$. The precisions of the variables are selected as 4 . The population size is selected as 40 . The crossover probability is selected as 0.75 and the mutation probability is selected as 0.05 . When the difference of the objective functional values between the best and the worst individuals in the population is less than 10-4, the algorithm is terminated. The obtained $\mathrm{Q}$ value is $\mathrm{Q}=0.002230$ and the transfer probability matrix of the state $\mathrm{P}^{\prime}$ is:

$$
P^{\prime}=\left[\begin{array}{cccc}
0.7261 & 0.0166 & 0.2573 & 0 \\
0.1467 & 0.7304 & 0 & 0.1229 \\
0.3427 & 0 & 0.6196 & 0.0377 \\
0.9956 & 0.0044 & 0 & 0
\end{array}\right]
$$

According to the obtained transfer probability matrix of the state, (1) is employed to predict the proportional values of the agriculture, forestry, animal husbandry and fishery industries in the Heilongjiang province between 2014 and 2015. The forecast is shown in Table 4.

\begin{tabular}{|c|c|c|c|c|c|c|c|c|c|c|c|}
\hline & $\begin{array}{c}\text { industri } \\
\text { es }\end{array}$ & $\begin{array}{c}200 \\
6\end{array}$ & $\begin{array}{c}200 \\
7\end{array}$ & $\begin{array}{c}200 \\
8\end{array}$ & 2009 & 2010 & $\begin{array}{c}201 \\
1\end{array}$ & $\begin{array}{c}201 \\
2\end{array}$ & $\begin{array}{c}201 \\
3\end{array}$ & $\begin{array}{c}201 \\
4\end{array}$ & $\begin{array}{c}201 \\
5 \\
\end{array}$ \\
\hline \multirow{8}{*}{ 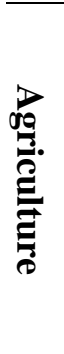 } & actual & 0.55 & 0.56 & 0.57 & 0.58 & 0.54 & 0.54 & 0.55 & 0.57 & & \\
\hline & value & 79 & 38 & 64 & 51 & 89 & 67 & 11 & 00 & & \\
\hline & forecast & 0.56 & 0.55 & 0.56 & 0.56 & 0.56 & 0.55 & 0.55 & 0.56 & 0.56 & 0.56 \\
\hline & value & 60 & 97 & 26 & 83 & 76 & 60 & 81 & 06 & 06 & 30 \\
\hline & absolute & 0.00 & 0.00 & 0.01 & 0.01 & 0.01 & 0.00 & 0.00 & 0.00 & & \\
\hline & error & 81 & 41 & 38 & 68 & 87 & 93 & 70 & 94 & & \\
\hline & relative & 1.45 & 0.73 & 2.39 & 2.87 & 3.41 & 1.70 & 1.27 & 1.65 & & \\
\hline & error & $\%$ & $\%$ & $\%$ & $\%$ & $\%$ & $\%$ & $\%$ & $\%$ & & \\
\hline \multirow{8}{*}{ 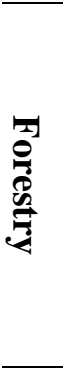 } & actual & 0.05 & 0.05 & 0.04 & 0.04 & 0.04 & 0.03 & 0.03 & 0.03 & & \\
\hline & value & 92 & 28 & 98 & 76 & 31 & 86 & 85 & 49 & & \\
\hline & forecast & 0.05 & 0.05 & 0.04 & 0.04 & 0.04 & 0.04 & 0.03 & 0.03 & 0.03 & 0.03 \\
\hline & value & 87 & 26 & 80 & 60 & 45 & 07 & 74 & 73 & 73 & 67 \\
\hline & absolute & 0.00 & 0.00 & 0.00 & 0.00 & 0.00 & 0.00 & 0.00 & 0.00 & & \\
\hline & error & 05 & 02 & 18 & 16 & 14 & 21 & 11 & 24 & & \\
\hline & relative & 0.84 & 0.38 & 3.61 & 3.36 & 3.25 & 5.44 & 2.86 & 6.88 & & \\
\hline & error & $\%$ & $\%$ & $\%$ & $\%$ & $\%$ & $\%$ & $\%$ & $\%$ & & \\
\hline \multirow{8}{*}{ 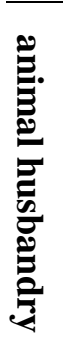 } & actual & 0.36 & 0.36 & 0.35 & 0.35 & 0.39 & 0.39 & 0.38 & 0.37 & & \\
\hline & value & 04 & 18 & 18 & 22 & 07 & 42 & 88 & 65 & & \\
\hline & forecast & 0.35 & 0.36 & 0.36 & 0.36 & 0.36 & 0.38 & 0.38 & 0.38 & 0.38 & 0.38 \\
\hline & value & 45 & 69 & 93 & 63 & 88 & 33 & 49 & 27 & 27 & 13 \\
\hline & absolute & 0.00 & 0.00 & 0.01 & 0.01 & 0.02 & 0.01 & 0.00 & 0.00 & & \\
\hline & error & 59 & 51 & 75 & 41 & 19 & 09 & 39 & 62 & & \\
\hline & relative & 1.64 & 1.41 & 4.97 & 4.00 & 5.61 & 2.77 & 1.00 & 1.65 & & \\
\hline & error & $\%$ & $\%$ & $\%$ & $\%$ & $\%$ & $\%$ & $\%$ & $\%$ & & \\
\hline \multirow{2}{*}{ 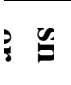 } & actual & 0.02 & 0.02 & 0.02 & 0.01 & 0.01 & 0.02 & 0.02 & 0.01 & & \\
\hline & & 25 & 15 & 20 & 51 & 73 & 05 & 16 & 86 & & \\
\hline
\end{tabular}

Table 4. Forecast Result and Forecast Precision 


\begin{tabular}{lcccccccccc} 
forecast & 0.02 & 0.02 & 0.02 & 0.01 & 0.01 & 0.02 & 0.01 & 0.01 & 0.01 & 0.01 \\
value & 09 & 09 & 01 & 94 & 91 & 00 & 96 & 94 & 94 & 90 \\
absolute & 0.00 & 0.00 & 0.00 & 0.00 & 0.00 & 0.00 & 0.00 & 0.00 & & \\
error & 16 & 06 & 19 & 43 & 18 & 05 & 20 & 08 & & \\
relative & 7.11 & 2.79 & 8.64 & 28.4 & 10.4 & 2.44 & 9.26 & 4.30 & & \\
error & $\%$ & $\%$ & $\%$ & $8 \%$ & $0 \%$ & $\%$ & $\%$ & $\%$ & & \\
\hline
\end{tabular}

According to the data listed in Table 4, by using our proposed improved genetic algorithm based on the Markov prediction model, the transfer probability matrix of the state of the structural value of the agriculture industry in the Heilongjiang province exhibits a higher prediction precision. The average relative error is $4.33 \%$.

\section{Conclusion}

The transfer probability matrix of the state based on a Markov prediction model is studied in this paper. By considering the relationships among the agriculture, forestry, animal husbandry and fishery industries of SMEs, a least square method is used to construct the Markov prediction model. The Markov prediction method is adopted to forecast the structural values of the agriculture, forestry, animal husbandry and fishery industries of SMEs in the Heilongiiang province between 2014 and 2015. The prediction results on the optimization of the structure of the agriculture industry in the Heilongjiang province are very useful. Meanwhile, they can be employed for the formulation of rational and effective policies for developing a structure of the agriculture industry. The result shows that the proposed improved genetic algorithm generates a good quality of population and it can be used for solving the transfer probability matrix of the state via a Markov model. It can overcome the shortcomings of existing methods such as statistical discrepancies, the requirement of large amount of calculations and complicated solving process. The proposed improved genetic algorithm for solving the transfer matrix of the state via the Markov model has high prediction precision. It is a practical method for short term forecasting applications.

\section{Acknowledgements}

This paper is supported by Philosophy and Social Scientific Research Project Foundation of Heilongjiang Province (No.14D008)and ((No.14C009). At the same time this paper is supported by the Higher Education Reform Project （No.JG2014010815）of Heilongjiang Province.

\section{References}

[1] A. M. Gonzalez, A. M. S. Roque and J. Garcia-Gonzalez, "Modeling and forecasting electricity prices with input/output hidden Markov models", IEEE Transactions on Power Systems, vol. 20, no. 1, (2012), pp. 13-24.

[2] L. Chen and K. Aihara, "Stability of genetic regulatory networks with time delay", IEEE Transactions on Circuits and Systems I, vol. 49, no. 5, (2012), pp. 602-608.

[3] Z. Wang, F. Yang, D. W. C. Ho, et al., "Stochastic dynamic modeling of short gene expression timeseries data", IEEE Transactions on Nanobioscience, vol. 7, no. 1, (2012), pp. 44-55.

[4] Y. Li, "Application on Markov Transfer Matrix Method in Predicting Enterprise Structure Rationality, Master dissertation", China Agricultural University, China, (2011).

[5] L. Zhenlie and J. Ling, "Applications of System Situation Transition Probability Matrix in Transportation Market”, Journal of Tongji University, vol. 30, no. 8, (2012), pp. 955-958.

[6] Z. Eryan and G. Wu, "A Kind of Statistical Method of Transition Probability Matrix Calculation", Hebei Vocational and Technical College Journal, vol. 3, no. 3, (2013).

[7] J. L. Liang, J. Lam and Z. D. Wang, "State estimation for Markov-type genetic regulatory networks with delays and uncertain mode transition rates", Physics Letters A, vol. 373, no. 47, (2012), pp. 4328-4337. 
[8] J. Xing, H. Yanji, Z. Mingliang, Y. Jifei and W. Junhua, "A Effective calcuation method of state transfer probability matrix of markov model", Journal of Projectiles, Rockets, Missiles and Guidance, (2013), pp. 244-249.

[9] L. Ximing, Z. Can and Y. Donghuang, "Genetic Algorithm Solving the Constrained Nonlinear Programming Problem Based On Species Selection”, Journal of Central South University Science and Technology, vol. 40, no. 1, (2013), pp. 185-189.

[10] G.-M. Yoon, J. Kim, Y.-H. Kim and B.-R. Moon, "Performance Improvement by Genetic Feature Selection and Adjusting Ratings' Mid-point Value in the Neural Network-Based Recommendation Models", Advances in information Sciences and Service Sciences (AISS), vol. 4, no. 11, (2012), pp. 3743.

[11] X. Ning and C. Chen, "Complementing the Two Intelligent Bionic Optimization Algorithms to Solve Construction Site Layout Problem", International Journal of Advancements in Computing Technology(IJACT), vol. 4, no. 1, (2012), pp. 1-14.

[12] W. Dingwei, W. Junwei, W. Hongfeng, Z. Ruiyou and G. Zhe, "Intelligent optimization methods", Beijing: Higher Education Press, (2012), pp. 20-55.

[13] J. E. Feng, J. Lam and Z. Shu, "Stabilization of Markovian systems via probability rate synthesis and output feedback", IEEE Transactions on Automatic Control, vol. 55, no. 3, (2010), pp. 773-777.

[14] L. Songsong, "Stock price time series prediction based on hidden markov model and computational intelligence", Doctoral dissertation, Harbin Institute of Technology, (2011).

[15] Y. Zhou and H. Fan, "The Application of Markov Skeleton Process on the Population Forecast", Journal of Convergence Information Technology(JCIT), vol. 7, no. 19, (2012), pp. 373-380.

[16] W. Fulin, "Agriculture systems engineering", Beijing: China agriculture press, (2012), pp. 147-148.

[17] W. Fulin, W. Changyou and S. Limin, "Research on forecasting the structure of agricultural production value, Agricultural System Engineering Theory and Practice Research”, Proceedings of the National Agricultural Systems Engineering Symposium, (2013), pp. 171-176.

[18] Z. Menglin, "Research on optimization the structure of agricultural industry of Heilongjiang province", Doctoral dissertation, Dongbei University of Finance and Economics, (2012).

[19] "The National Bureau of Statistics of the People's Republic of China compiles", China Statistical Yearbook. Beijing: China Statistical Publishing House, (2003-2012).

[20] R. Banker and C. A. Cooper, "Models for Estimating Technical and Scale Inefficiencies in Data Envelopment Analysis", Management Science, no. 30, (2011), pp. 1078-1092.

[21] G. Chow and A.-L. Lin, "Accounting for Economic Growth in Taiwan and Mainland China: A Comparative Analysis", Journal of Comparative Economics, vol. 30, Issue 3, (2012), pp. 507-530.

[22] A. Charnes, W. Cooper and B. Golang, Journal of Econometrics, Foundations of Data Envelopment Analysis for Pareto-Koopmans Efficient Empirical Production Functions, no. 30, (2009), pp. 0304-4076.

[23] B. M. Fleisher and J. Chen, "The Coast-noncoast Income Gap, Productivity and Regional Economic Policy in China", Journal of Comparative Economics, no. 25, (2012), pp. 220-236.

[24] G. H. Jefferson, T. G. Rawski and Y. Zheng, "Growth, Efficiency and Convergence in China's State and Collective Industry”, Economic Development and Cultural Change, no. 40, (2012), pp. 239-266.

[25] K. P. Kalirajan, M. B. Obwona and S. Zhao, "A Decomposition of Total Factor Productivity Growth: The Case of China's Agricultural Growth Before and After Reform", American Journal of Agricultural Economics, vol. 78, no. 2, (2012), pp. 331-338

\section{Author}

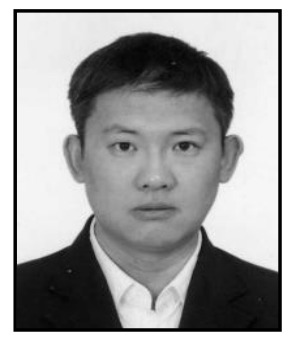

Wei Chuanli was born in July 1975 in the Harbin of Hei Longjiang Province. He graduated from the Harbin Normal University with a B.A. in 1998. He then pursued further postgraduate study in the Management Department of HIT University and graduated in 2005 with a M.A. He succeeded in passing the Project Management Professional (PMP) credential examination; PMP (project management professional) 212202, PMI membership (project management institute530902) in 2004.12.8. He was awarded the academic title as associate professor in September 2008. He was recruited by the Ministry of Education and worked as a Doctor student in the UTC of France from July 2008 to December 2010, majored in EGovernment. He then specialized in Systems Engineering in 
Northeast Agricultural University and graduated with a Doctor's degree of Management in June 2011. He has completed writing 18 academic papers, and 4 of them were published in CSSCI Journals. He published a book written by him, edited or co-edited 5 books and presided over a research project supervised by Hei Longjiang Provincial Bureau of Education. 